\title{
Asymptotic soliton train solutions of the defocusing nonlinear Schrödinger equation
}

\author{
A. M. Kamchatnov, ${ }^{1,2}$ R. A. Kraenkel, ${ }^{2}$ and B. A. Umarov ${ }^{2,3}$ \\ ${ }^{1}$ Institute of Spectroscopy, Russian Academy of Sciences, Troitsk 142190, Moscow Region, Russia \\ ${ }^{2}$ Instituto de Física Teórica, Universidade Estadual Paulista-UNESP, Rua Pamplona 145, 01405-900 São Paulo, Brazil \\ ${ }^{3}$ Physical-Technical Institute, Uzbek Academy of Sciences, 700084 Tashkent-84, G. Mavlyanov Street, 2-b, Uzbekistan \\ (Received 23 October 2001; revised manuscript received 21 February 2002; published 20 September 2002)
}

\begin{abstract}
Asymptotic behavior of initially "large and smooth" pulses is investigated at two typical stages of their evolution governed by the defocusing nonlinear Schrödinger equation. At first, wave breaking phenomenon is studied in the limit of small dispersion. A solution of the Whitham modulational equations is found for the case of dissipationless shock wave arising after the wave breaking point. Then, asymptotic soliton trains arising eventually from a large and smooth initial pulse are studied by means of a semiclassical method. The parameter varying along the soliton train is calculated from the generalized Bohr-Sommerfeld quantization rule, so that the distribution of eigenvalues depends on two functions - intensity $\rho_{0}(x)$ of the initial pulse and its initial chirp $v_{0}(x)$. The influence of the initial chirp on the asymptotic state is investigated. Excellent agreement of the numerical solution of the defocusing NLS equation with predictions of the asymptotic theory is found.
\end{abstract}

DOI: 10.1103/PhysRevE.66.036609

PACS number(s): 42.65.Tg

\section{INTRODUCTION}

Nonlinear Schrödinger (NLS) equation is a universal equation that describes the evolution of envelopes of linear waves under the influence of weak dispersion and nonlinear effects in a variety of physical systems. Depending on the sign of the nonlinear term in this equation, the focusing and defocusing cases with quite different properties are distinguished. In particular, the focusing NLS equation has bright soliton solutions propagating on the zero background, whereas defocusing NLS equation does not support bright solitons and instead has dark soliton solutions on the nonzero plane wave oscillating background [1].

We shall consider here the defocusing NLS equation with initial conditions in the form of a large and smooth pulse described by the intensity $\rho_{0}(x)$ and chirp $v_{0}(x)$. It is known that in the limit of negligible dispersion a general enough initial dark pulse governed by the defocusing NLS equation evolves at some critical time to the formation of the wave breaking point and taking into account the small dispersion leads to the onset of oscillations just after the wave breaking point $[2,3]$. The region of oscillations arising here can be presented as a modulated periodic solution of the NLS equation. This permits one to apply to this problem the Whitham theory of modulations $[4,5]$ which was used previously in the solution of similar problems about the evolution of large pulses described by the Korteweg-de Vries (KdV) equation [6-9]. Generalization of the Whitham theory on the defocusing NLS equation case was developed in Refs. [10-13]. In a general case the solution of the Whitham equations is quite complicated. However, in the vicinity of the wave breaking point the pulse can be described by simplified approximate formulas which admit an exact analytical solution of the Whitham equations in a closed form. For the case of the KdV equation such a solution was found in Refs. $[14,15]$. Here we shall find a similar solution of Whitham equations for the NLS equation case that describes the so-called dissipationless shock wave arising after the wave breaking point.

Further evolution of the pulse leads to the stage when oscillations occupy the whole pulse and they evolve eventually into a train of solitons. As was noticed in Refs. [16,17] for the $\mathrm{KdV}$ equation case, the parameters of these asymptotic solitons can be calculated from a semiclassical treatment of the quantum mechanical Schrödinger operator associated with the $\mathrm{KdV}$ equation in the framework of the inverse scattering transform method with a potential determined by the initial data. Then, for a large pulse leading to a great number of solitons at the final stage, the spectrum can be found with the use of the semiclassical (BohrSommerfeld) quantization rule that gives a full description of the asymptotic stage of the evolution. The theory developed in Refs. [12] shows that a similar approach applies also to the defocusing NLS equation. Here we shall obtain the necessary generalization of the Bohr-Sommerfeld quantization rule by a simple method $[18,19]$ which applies also to many other integrable wave equations. The results obtained permit us to estimate the influence of initial chirp on the evolution of large pulses in optical fibers $[2,11,20,21]$ and other nonlinear materials, e.g. magnetic films [22,23].

\section{PERIODIC AND DARK SOLITON SOLUTIONS OF THE DEFOCUSING NLS EQUATION}

We shall consider the defocusing NLS equation in the standard dimensionless form

$$
i \varepsilon u_{t}+\varepsilon^{2} u_{x x}-2|u|^{2} u=0,
$$

where $u(x, t)$ is the envelope amplitude evolving with time $t$ during the propagation of the pulse along $x$ axis and small parameter $\varepsilon \ll 1$ controls the relative magnitude of the dispersion effects. This equation is completely integrable, that is, it can be presented as a compatibility condition of two linear systems [24] $\psi_{x}=U \psi, \psi_{t}=V \psi$, where $U$ and $V$ are $2 \times 2$ matrices. However, for the investigation of a semiclassical limit it is more convenient to use a scalar representation $[19,25]$ in the form

$$
\varepsilon^{2} \psi_{x x}=\mathcal{A} \psi, \quad \psi_{t}=-\frac{1}{2} \mathcal{B}_{x} \psi+\mathcal{B} \psi_{x},
$$


where

$$
\begin{gathered}
\mathcal{A}=-\left(\lambda+\frac{i \varepsilon}{2} \frac{u_{x}}{u}\right)^{2}+|u|^{2}-\frac{\varepsilon^{2}}{2}\left(\frac{u_{x x}}{u}-\frac{u_{x}^{2}}{u^{2}}\right), \\
\mathcal{B}=-2 \lambda+\frac{i \varepsilon u_{x}}{u},
\end{gathered}
$$

which was first obtained in Ref. [26]. The transition to semiclassical limit is achieved by means of the substitution

$$
u(x, t)=\sqrt{\rho(x, t)} \exp \left(\frac{i}{\varepsilon} \int^{x} v\left(x^{\prime}, t\right) d x^{\prime}\right)
$$

so that NLS Eq. (1) transforms into a system of equations for intensity $\rho(x, t)$ and chirp $v(x, t)$ :

$$
\frac{1}{2} \rho_{t}+(\rho v)_{x}=0, \frac{1}{2} v_{t}+v v_{x}+\rho_{x}+\varepsilon^{2}\left(\rho_{x}^{2} / 8 \rho^{2}-\rho_{x x} / 4 \rho\right)_{x}=0 .
$$

Thus, in the semiclassical limit $\varepsilon \rightarrow 0$ the initial pulse at $t$ $=0$ is determined by smooth distributions of $\rho_{0}(x)$ and $v_{0}(x)$, whereas $u_{0}(x)$ may be a fast oscillating function if $v_{0}(x) \neq 0$. We are interested in finding solutions at typical stages of pulse evolution.

As was noticed above, we suppose that arising after the wave breaking point, the regions of oscillations can be presented as modulated periodic waves-soliton trains. Such periodic solutions of Eq. (1) can be obtained as follows. It is easy to check that the functions

$$
\psi^{ \pm}=\sqrt{g} \exp \left( \pm \frac{i}{\varepsilon} \int \frac{x \sqrt{P(\lambda)}}{g} d x\right)
$$

satisfy the first part of Eq. (2) provided that $g(x, t)$ satisfies the equation

$$
\varepsilon^{2}\left(\frac{1}{2} g g_{x x}-\frac{1}{4} g_{x}^{2}\right)-\mathcal{A} g^{2}=P(\lambda),
$$

where the constant on the right-hand side can only depend on the spectral parameter $\lambda$. The periodic solution is distinguished by the condition that $P(\lambda)$ be a polynomial in $\lambda$, and the one-phase periodic solution corresponds to the fourth degree polynomial

$$
P(\lambda)=\prod_{i=1}^{4}\left(\lambda-\lambda_{i}\right)=\lambda^{4}-s_{1} \lambda^{3}+s_{2} \lambda^{2}-s_{3} \lambda+s_{4} .
$$

Then $g(x, t)$ is the first degree polynomial

$$
g(x, t)=\mu(x, t)-\lambda,
$$

and by taking $\lambda=\mu$ in Eq. (7) we obtain the equation for $\mu(x, t)$,

$$
\varepsilon \mu_{x}=2 \sqrt{-P(\mu)} .
$$

From the second part of Eq. (2) we find that $g(x, t)$ satisfies also the equation

$$
g_{t}=\mathcal{B} g_{x}-\mathcal{B}_{x} g
$$

which in a similar way yields

$$
\mu_{t}=-s_{1} \mu_{x},
$$

where we have used the identity

$$
\mu(x, t)=s_{1} / 2+i \varepsilon u_{x} / 2 u
$$

following from Eq. (7) after equating coefficients of $\lambda^{3}$ on both sides of this formula. Thus, $\mu(x, t)$ depends only on the phase $\xi=x-s_{1} t$ and obeys the equation

$$
\varepsilon \frac{d \mu}{d \xi}=2 \sqrt{-P(\mu)}, \quad \xi=x-s_{1} t
$$

The variable $\mu(x, t)$ is complex and moves along a certain curve in the complex plane determined by the so-called "reality conditions" (see Ref. [5]). These conditions distinguish physically meaningful solutions of Eq. (14) and lead to the following expression for the intensity of the nonlinear periodic wave:

$$
\begin{aligned}
\rho(x, t)= & |u(x, t)|^{2}=\frac{1}{4}\left(\lambda_{1}-\lambda_{2}-\lambda_{3}+\lambda_{4}\right)^{2} \\
& +\left(\lambda_{1}-\lambda_{2}\right)\left(\lambda_{3}-\lambda_{4}\right) \\
& \times \operatorname{sn}^{2}\left[\sqrt{\left(\lambda_{1}-\lambda_{3}\right)\left(\lambda_{2}-\lambda_{4}\right)} \xi / \varepsilon, m\right],
\end{aligned}
$$

where the zeros $\lambda_{i}, i=1,2,3,4$, are ordered according to

$$
\lambda_{1} \geqslant \lambda_{2} \geqslant \lambda_{3} \geqslant \lambda_{4},
$$

the parameter $m$ of the elliptic function is defined by

$$
m=\frac{\left(\lambda_{1}-\lambda_{2}\right)\left(\lambda_{3}-\lambda_{4}\right)}{\left(\lambda_{1}-\lambda_{3}\right)\left(\lambda_{2}-\lambda_{4}\right)}
$$

and

$$
\xi=x-V t, \quad V=s_{1}=\sum_{i=1}^{4} \lambda_{i}
$$

$V$ being the phase velocity of the nonlinear wave.

In a weakly modulated nonlinear periodic wave the parameters $\lambda_{i}, i=1,2,3,4$, become slow functions of $x$ and $t$ which change little in one wavelength and one period. The evolution of $\lambda_{i}$ is governed by the Whitham modulation equations (obtained for the NLS equation case in Refs. $[27,28])$

$$
\frac{\partial \lambda_{i}}{\partial t}+v_{i}(\lambda) \frac{\partial \lambda_{i}}{\partial x}=0, \quad i=1,2,3,4
$$

where the Whitham velocities are equal to

$$
\begin{gathered}
v_{i}(\lambda)=\left(1-\frac{L}{\partial_{i} L} \partial_{i}\right) V, \quad \partial_{i} \equiv \partial / \partial \lambda_{i}, \quad i=1,2,3,4, \\
V=\sum \lambda_{i},
\end{gathered}
$$


and $L(\lambda)$ is a wavelength

$$
L=\frac{K(m)}{\sqrt{\left(\lambda_{1}-\lambda_{4}\right)\left(\lambda_{2}-\lambda_{3}\right)}},
$$

$K(m)$ being the complete elliptic integral of the first kind. Substitution of Eq. (21) into Eq. (20) gives the Whitham velocities in the form

$$
\begin{aligned}
& v_{1}=\sum \lambda_{i}+\frac{2\left(\lambda_{1}-\lambda_{2}\right)\left(\lambda_{1}-\lambda_{4}\right) K}{\left(\lambda_{1}-\lambda_{4}\right) K-\left(\lambda_{2}-\lambda_{4}\right) E}, \\
& v_{2}=\sum \lambda_{i}-\frac{2\left(\lambda_{1}-\lambda_{2}\right)\left(\lambda_{2}-\lambda_{4}\right) K}{\left(\lambda_{2}-\lambda_{3}\right) K-\left(\lambda_{1}-\lambda_{3}\right) E}, \\
& v_{3}=\sum \lambda_{i}+\frac{2\left(\lambda_{2}-\lambda_{3}\right)\left(\lambda_{3}-\lambda_{4}\right) K}{\left(\lambda_{2}-\lambda_{3}\right) K-\left(\lambda_{2}-\lambda_{4}\right) E}, \\
& v_{4}=\sum \lambda_{i}-\frac{2\left(\lambda_{1}-\lambda_{3}\right)\left(\lambda_{3}-\lambda_{4}\right) K}{\left(\lambda_{1}-\lambda_{4}\right) K-\left(\lambda_{1}-\lambda_{3}\right) E},
\end{aligned}
$$

where $K=K(m)$ and $E=E(m)$ are complete elliptic integrals of the first and second kind, respectively. In this case the parameters $\lambda_{i}$ are called Riemann invariants of the Whitham equations and our first task is to find their solution corresponding to the region of oscillations arising after the wave breaking point. At one edge of this region we have $m$ $=1$ or $\lambda_{3}=\lambda_{2}$, so that the intensity distribution is given by

$$
\rho_{s}(x, t)=\frac{1}{4}\left(\lambda_{1}-\lambda_{4}\right)^{2}-\frac{\left(\lambda_{1}-\lambda_{2}\right)\left(\lambda_{2}-\lambda_{4}\right)}{\cosh ^{2}\left[\sqrt{\left(\lambda_{1}-\lambda_{2}\right)\left(\lambda_{2}-\lambda_{4}\right)} \xi / \varepsilon\right]},
$$

where $\xi=x-\left(\lambda_{1}+2 \lambda_{2}+\lambda_{4}\right) t$, and at the other side with $m=0$ where $\lambda_{2}=\lambda_{1}$ we obtain a smooth solution

$$
\rho(x, t)=\frac{1}{4}\left(\lambda_{3}-\lambda_{4}\right)^{2} .
$$

Thus, in the problem of the evolution of dissipationless shock wave we have to find boundaries of the region of oscillations and the dependence of the parameters $\lambda_{i}$ on $x$ and $t$ within it.

At the asymptotically large values of time the pulse evolves into a train of dark soliton solutions with $m=1$ [Eq. (23)]. The values of $\lambda_{1}$ and $\lambda_{4}$ are determined by the background wave and hence are constant, so that the parameters of solitons in the train are determined by the values of $\lambda_{2}$. Our second task is to find how $\lambda_{2}$ varies along the soliton train arising from the initial pulse with given distributions of $\rho_{0}(x)$ and $v_{0}(x)$.

\section{DISSIPATIONLESS SHOCK WAVE}

For smooth pulses, when we can neglect higher space derivatives in Eqs. (5), we arrive at equations of hydrodynamical type,

$$
\frac{1}{2} \rho_{t}+(\rho v)_{x}=0, \quad \frac{1}{2} v_{t}+v v_{x}+\rho_{x}=0
$$

which correspond to the dispersionless limit of the NLS equation (1). It is convenient to introduce variables called Riemann invariants,

$$
\lambda_{ \pm}=\frac{1}{2} v \pm \sqrt{\rho}
$$

so that Eqs. (25) take a convenient symmetric form

$$
\frac{\partial \lambda_{+}}{\partial t}+\left(3 \lambda_{+}+\lambda_{-}\right) \frac{\partial \lambda_{+}}{\partial x}=0, \quad \frac{\partial \lambda_{-}}{\partial t}+\left(\lambda_{+}+3 \lambda_{-}\right) \frac{\partial \lambda_{-}}{\partial x}=0 .
$$

Initial data are given by two functions $\lambda_{+}(x, 0)$ and $\lambda_{-}(x, 0)$ determined by the initial distributions $\rho_{0}(x)$ and $v_{0}(x)$. The system (27) leads to two families of characteristics, i.e. lines in the $(x, t)$ plane along which one of the two Riemann invariants (either $\lambda_{+}$or $\lambda_{-}$) is constant. The wave breaking point corresponds to the moment when characteristics of one of the families begin to intersect each other, so that the corresponding Riemann invariant becomes a three-valued function of the space coordinate. Let such an intersection occur for the characteristics with constant $\lambda_{+}$. Then at the wave breaking point the profile of $\lambda_{+}$as a function of $x$ has a vertical tangent line and, hence, in the vicinity of this point it varies very fast, whereas the second Riemann invariant varies with $x$ much slower and may be considered here as a constant parameter,

$$
\lambda_{-}=\lambda_{0}=\text { const. }
$$

Thus, the second part of Eq. (27) is solved by Eq. (28) and the first part of Eq. (27) has a well-known solution

$$
x-\left(3 \lambda_{+}+\lambda_{0}\right) t=f\left(\lambda_{+}\right) .
$$

Since at the wave breaking time moment $t=0$ the function $x=f\left(\lambda_{+}\right)$must have an inflection point with a vertical tangent line, in the vicinity of this point $f\left(\lambda_{+}\right)$can be approximated by a cubic function,

$$
x-\left(3 \lambda_{+}+\lambda_{0}\right) t=-C\left(\lambda_{+}-\bar{\lambda}_{+}\right)^{3} .
$$

Equations (28) and (29) (at $t=0$ ) may be considered as initial data for the Riemann invariants in the vicinity of the wave breaking point.

The "shallow water equations" (25) are invariant with respect to the Galileo transformation,

$$
\begin{gathered}
x^{\prime}=x-2 v_{0} t, \quad t^{\prime}=t, \quad \rho=\rho^{\prime}, \quad v=v^{\prime}+v_{0}, \\
\lambda_{ \pm}=\lambda_{ \pm}^{\prime}+\frac{1}{2} v_{0},
\end{gathered}
$$

and scaling transformation

$$
x=a x^{\prime}, \quad t=t^{\prime}, \quad \rho=a^{2} \rho^{\prime}, \quad v=a v^{\prime}, \quad \lambda_{ \pm}=a \lambda_{ \pm}^{\prime},
$$

which allow us to transform Eqs. (28) and (29) to a simple form

$$
x-\left(3 \lambda_{+}+\bar{\lambda}\right) t=-\lambda_{+}^{3}, \quad \lambda_{-}=\bar{\lambda}=\mathrm{const},
$$




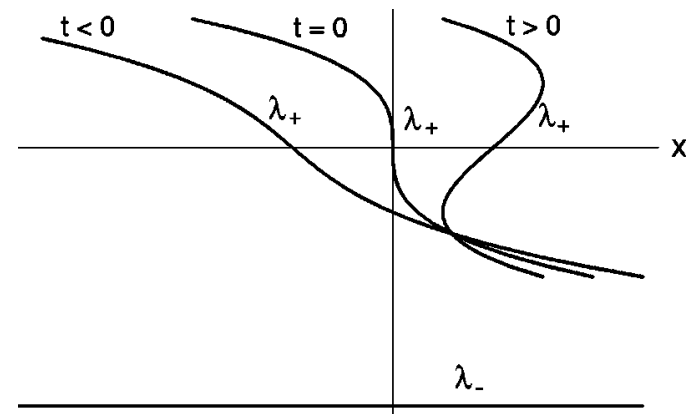

FIG. 1. The dependence of Riemann invariants $\lambda_{ \pm}$on $x$ at different moments of time.

where we use the previous notation (without primes) for the Riemann invariants and space and time coordinates. Thus, in the dispersionless limit the solution of Eqs. (27) has the form (30) in the vicinity of the wave breaking point (see Fig. 1).

The formation of a multivalued region of $\lambda_{+}(x)$ shows that we cannot neglect dispersion in the vicinity of this point. The dispersion effects lead to the formation of the region of oscillations where the solution of the NLS equation (1) can be approximated by a modulated periodic wave. In our approximation we suppose that $\lambda_{4}$ is constant within the region of oscillations and $\lambda_{1}, \lambda_{2}$, and $\lambda_{3}$ vary so that at one of its boundaries we have $\lambda_{2}=\lambda_{3}(m=1)$ and at the other boundary $\lambda_{2}=\lambda_{1}(m=0)$. Correspondingly, the Whitham velocities (22) are equal at these limits to

$$
v_{1}=3 \lambda_{1}+\lambda_{4}, \quad v_{2}=v_{3}=\sum \lambda_{i}, \quad v_{4}=\lambda_{1}+3 \lambda_{4}
$$

at $\lambda_{2}=\lambda_{3}(m=1)$, and to

$$
\begin{aligned}
v_{1}=v_{2} & =\sum \lambda_{i}+\frac{4\left(\lambda_{1}-\lambda_{3}\right)\left(\lambda_{1}-\lambda_{4}\right)}{2 \lambda_{1}-\lambda_{3}-\lambda_{4}}, v_{3}=3 \lambda_{3}+\lambda_{4}, \\
v_{4} & =\lambda_{3}+3 \lambda_{4}
\end{aligned}
$$

at $\lambda_{2}=\lambda_{1}(m=0)$. Hence, two of the four Whitham equations (19) reduce to Eqs. (27) at the boundaries of the region of oscillations, and therefore the corresponding Riemann invariants have to coincide with $\lambda_{ \pm}$at these boundaries,

$$
\begin{gathered}
\lambda_{1}=\lambda_{+} \quad \text { at } m=1, \quad \lambda_{3}=\lambda_{+} \text {at } m=0, \\
\text { and } \lambda_{4}=\lambda_{-} \text {in both cases. }
\end{gathered}
$$

Thus we have to find such a solution of Eqs. (19) which gives

$$
\begin{aligned}
& x-\left(3 \lambda_{1}+\lambda_{4}\right) t=-\lambda_{1}^{3} \quad \text { at } \quad m=1, \\
& x-\left(3 \lambda_{3}+\lambda_{4}\right) t=-\lambda_{3}^{3} \quad \text { at } \quad m=0 .
\end{aligned}
$$

In the generalized hodograph method [29] the solution is looked for in the form

$$
x-v_{i}(\lambda) t=w_{i}(\lambda), \quad i=1,2,3,4
$$

where $v_{i}(\lambda)$ are the Whitham velocities (20) and $w_{i}(\lambda)$ are velocities of a flow

$$
\frac{\partial \lambda_{i}}{\partial \tau}+w_{i}(\lambda) \frac{\partial \lambda_{i}}{\partial x}=0, \quad i=1,2,3,4
$$

commuting with Eqs. (19). If we represent $w_{i}(\lambda)$ in the form analogous to Eqs. (20),

$$
w_{i}(\lambda)=\left(1-\frac{L}{\partial_{i} L} \partial_{i}\right) W, \quad i=1,2,3,4,
$$

then the condition of commutativity of flows (19) and (36) reduces to the Euler-Poisson equations [10]

$$
\partial_{i} \partial_{j} W-\frac{1}{2\left(\lambda_{i}-\lambda_{j}\right)}\left(\partial_{i} W-\partial_{j} W\right)=0, \quad i \neq j .
$$

It is easy to check that this equation has a particular solution $W=$ const $/ \sqrt{P(\lambda)}, P(\lambda)=\Pi\left(\lambda-\lambda_{i}\right)$, which is sufficient for our aim. We choose the normalization factor so that the coefficient before $\lambda^{-1}$ in the series expansion of $W$ in powers of $\lambda^{-1}$ be equal to $s_{1}$ and, hence, the corresponding $w_{i}$ coincide with the Whitham velocities (20). Thus, we obtain a sequence of $W=W^{(k)}$ defined by the generating function

$$
\begin{gathered}
W=\frac{2 \lambda^{2}}{\sqrt{P(\lambda)}}=\sum \frac{W^{(k)}}{\lambda^{k}}=2+\frac{s_{1}}{\lambda}+\frac{\frac{3}{8} s_{1}^{2}-\frac{1}{2} s_{2}}{\lambda^{2}} \\
+\frac{\frac{5}{16} s_{1}^{3}-\frac{3}{4} s_{1} s_{2}+\frac{1}{2} s_{3}}{\lambda^{3}}+\cdots,
\end{gathered}
$$

where $P(\lambda)$ coincides with the polynomial in Eq. (8). Thus, a sequence of velocities of the commuting flows is given by

$$
w_{i}^{(k)}=W^{(k)}+\left(v_{i}-s_{1}\right) \partial_{i} W^{(k)}
$$

Then a simple calculation shows that the solution (35) satisfying the boundary conditions (34) has the form

$$
x-v_{i}(\lambda) t=-\frac{8}{35} w_{i}^{(3)}(\lambda)+\frac{4}{35} \bar{\lambda} w_{i}^{(2)}+\frac{1}{35} \bar{\lambda}^{2} v_{i}(\lambda)+\frac{1}{35} \bar{\lambda}^{3},
$$

$$
i=1,2,3 \text {; }
$$


These formulas give the solution of our problem in an implicit form.

Let us find the laws of motion of the boundaries of the oscillatory region. At $m=1$ Eqs. (41) with $i=1$ and $i=3$ give

$$
\begin{gathered}
x-\left(3 \lambda_{1}+\bar{\lambda}\right) t=-\lambda_{1}^{3}, \\
x-\left(\lambda_{1}+2 \lambda_{3}+\bar{\lambda}\right) t=-\frac{1}{35}\left(5 \lambda_{1}^{3}+6 \lambda_{1}^{2} \lambda_{3}+8 \lambda_{1} \lambda_{3}^{2}+16 \lambda_{3}^{3}\right),
\end{gathered}
$$

and their difference gives the relation

$$
t=\frac{1}{35}\left(15 \lambda_{1}^{2}+12 \lambda_{1} \lambda_{3}+8 \lambda_{3}^{2}\right) .
$$

The other two equations of Eq. (41) expanded into series in powers of $\lambda_{2}^{\prime}=\lambda_{2}-\lambda_{3}$ yield after subtraction another relation

$$
t=\frac{1}{35}\left(3 \lambda_{1}^{2}+8 \lambda_{1} \lambda_{3}+24 \lambda_{3}^{2}\right) .
$$

Equating (42) and (43) to each other, we find at this boundary the relation

$$
\lambda_{3}=-\frac{3}{4} \lambda_{1},
$$

which coincides with an analogous relation in the $\mathrm{KdV}$ equation case $[5,14]$. Then from Eqs. (42) or (43) we obtain at the left boundary $t=\frac{3}{10} \lambda_{1}^{2}$ and hence the left boundary of the region of oscillations moves according to the law

$$
x^{-}(t)=\left(3 \lambda_{1}+\bar{\lambda}\right) t-\lambda_{1}^{3}=\bar{\lambda} t-\frac{1}{3} \sqrt{\frac{10}{3}} t^{3 / 2} .
$$

Analogous calculation at $m=0\left(\lambda_{2}=\lambda_{1}\right)$ yields the relations

$$
\begin{gathered}
t=\frac{\left(8 \lambda_{1}-7 \bar{\lambda}\right)\left(8 \lambda_{1}^{2}+4 \lambda_{1} \lambda_{3}+3 \lambda_{3}^{2}\right)-15 \lambda_{3}^{3}}{35\left(4 \lambda_{1}-\lambda_{3}-3 \bar{\lambda}\right)} \\
21 \bar{\lambda}^{2}\left(4 \lambda_{1}+\lambda_{3}\right)-10 \bar{\lambda}\left(20 \lambda_{1}^{2}+2 \lambda_{1} \lambda_{3}+\lambda_{3}^{2}\right)+16 \lambda_{1}\left(8 \lambda_{1}^{2}\right. \\
\left.-\lambda_{1} \lambda_{3}-\lambda_{3}^{2}\right)+9 \lambda_{3}^{3}=0 .
\end{gathered}
$$

In the limit $|\bar{\lambda}| \rightarrow \infty$, these equation reproduce the relations for the $\mathrm{KdV}$ equation case $[5,14]$, in particular, $\lambda_{1}=-\frac{1}{4} \lambda_{3}$. However, in the NLS equation case the solution (41) is not self-similar and depends on the parameter $\bar{\lambda}$. An example of the dependence of the Riemann invariants $\lambda_{1}, \lambda_{2}, \lambda_{3}$ on $x$ at a fixed value of $t$ is shown in Fig. 2. Substitution of these functions $\lambda_{i}(x)$ into Eq. (15) gives the dependence of intensity on $x$ shown in Fig. 3. This plot illustrates the process of the formation of a shock for the NLS equation case.

\section{ASYMPTOTIC STAGE OF EVOLUTION}

In the asymptotic stage of the evolution the pulse decays into a set of solitons which can be presented as modulated one-phase periodic soliton trains. Each separate soliton is described by Eq. (23) and parameters $\lambda_{1}$ and $\lambda_{4}$ are constant along the train, whereas the parameter $\lambda_{2}$ changes its value from one soliton to another. Thus, the properties of the train

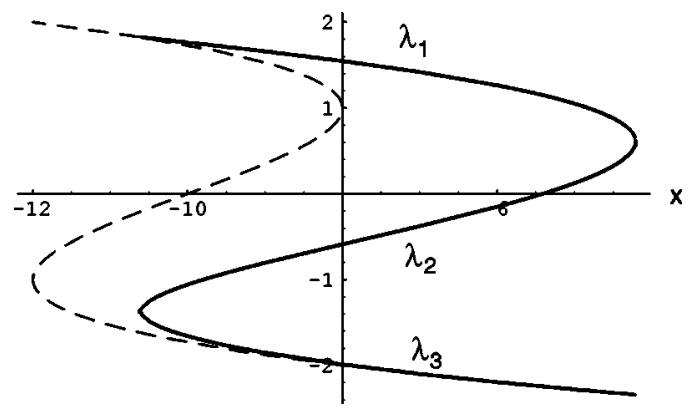

FIG. 2. The dependence of Riemann invariants $\lambda_{1}, \lambda_{2}, \lambda_{3}$ on $x$ at fixed value of time $t=1$. The fourth Riemann invariant $\lambda_{4}=\bar{\lambda}$ $=-10$ is not depicted. The dashed line shows the corresponding dependence of $\lambda_{+}$for the solution of equations in the dispersionless limit.

are determined by the spectrum of values $\lambda_{2}$. It is known that this spectrum coincides with the eigenvalues of the first part of Eq. (2) for given $\rho_{0}(x)$ and $v_{0}(x)$ and it does not change during the evolution of $u(x, t)$ governed by the NLS equation. In the case of large and smooth pulses with large number of eigenvalues, the spectrum can be determined, in principle, by means of a semiclassical treatment of the eigenvalue problem. The corresponding generalization of semiclassical quantization rules can be found with the use of a matrix (Zakharov-Shabat) spectral problem associated with the NLS equation (see, e.g., Refs. [12,30-32]). Here we shall show that it follows very simply from the scalar form (2), and this method of derivation can be generalized on many other integrable equations (see Refs. $[19,25]$ ).

For strictly periodic case, the solution of the second-order Eq. (2) is given by Eq. (6) with constant $P(\lambda)$. For a slowly modulated wave the zeros $\lambda_{i}$ become slow functions of $x$ and $t$, and for this reason we inserted $P(\lambda)$ under the integration sign in Eq. (6). With the use of Eqs. (7), (9), and (13), we can present $\psi^{ \pm}$in the form

$$
\begin{aligned}
\psi^{ \pm} \sim \exp \left( \pm \frac{i}{\varepsilon} \int \frac{x}{g}\left\{\left[\left(\lambda+i \varepsilon u_{x} / 2 u\right)^{2}-|u|^{2}\right.\right.\right. \\
\left.\left.\left.\quad+\varepsilon^{2}\left(u_{x x} / 2 u-u_{x}^{2} / 2 u^{2}\right)\right] g^{2}+\varepsilon^{2}\left(g g_{x x} / 2-g_{x}^{2} / 4\right)\right\}^{1 / 2} d x\right),
\end{aligned}
$$

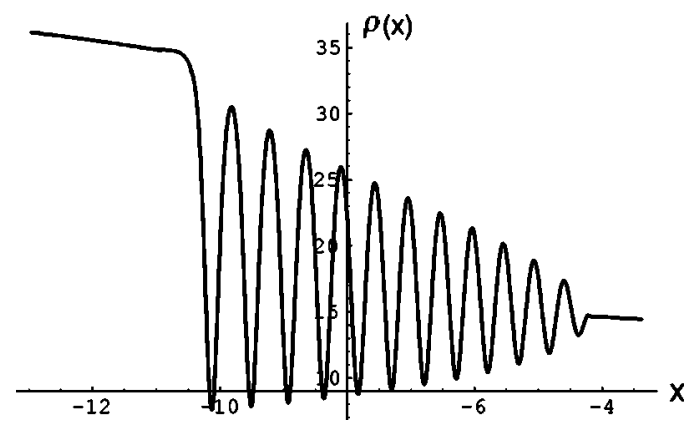

FIG. 3. Dissipationless shock wave for the NLS equation case. The plot corresponds to the time $t=1$ and $\lambda_{4}=\bar{\lambda}=-10$, and $\varepsilon$ $=1$. 
where

$$
g=s_{1} / 2+i \varepsilon u_{x} / 2 u-\lambda .
$$

We suppose that this expression holds approximately for all moments of time including the initial state. Then, since the initial distributions of $\rho_{0}(x)=|u(x, 0)|^{2}$ and $v_{0}(x)=$ $-i \varepsilon u_{x} / u$ are supposed to be smooth functions of $x$, we can neglect the derivatives of $v_{0}(x)$ in the weak dispersion limit $\varepsilon^{2} \ll 1$ to obtain the WKB wave function of the initial state,

$$
\psi_{W K B} \sim \exp \left( \pm \frac{i}{\varepsilon} \int^{x} \sqrt{\left[\lambda-\frac{1}{2} v_{0}(x)\right]^{2}-\rho_{0}(x)} d x\right) .
$$

The eigenvalues $\lambda$ are determined in this approximation by the well-known Bohr-Sommerfeld quantization rule [33]

$$
\begin{gathered}
\frac{1}{\varepsilon} \oint p(x) d x=2 \pi\left(n+\frac{1}{2}\right), \quad n=0,1,2, \ldots, N, \\
p(x)=\sqrt{\left[\lambda-\frac{1}{2} v_{0}(x)\right]^{2}-\rho_{0}(x),}
\end{gathered}
$$

where the integration is taken over the cycle around two turning points where the integrand function vanishes. Eigenvalues found in this way are equal to the values of the parameter $\lambda_{2}$ in soliton solution (23) when solitons are well separated from each other in the asymptotic soliton train.

It is instructive to note that the rule (51) corresponds to the semiclassical quantization of a mechanical system with the Hamiltonian

$$
H(p, x)=\left[\sqrt{p^{2}+\rho_{0}(x)}-\frac{1}{2} v_{0}(x)\right]^{2},
$$

where $x$ is a coordinate and $p$ a momentum. At the turning points the momentum $p$ vanishes. Equation (51) states that the area inside the contour $H(p, x)=$ const in the phase plane $(x, p)$ can only take half-integer values in units of $2 \pi \varepsilon$.

The regions of the possible values of $\lambda$ are determined by the condition that the expression under the square root in Eq. (51) is positive and has two real turning points. Thus the plots of the "potentials" (Riemann invariants in the zero dispersion limit)

$$
\lambda_{ \pm}(x)=\frac{1}{2} v_{0}(x) \pm \sqrt{\rho_{0}(x)}
$$

permit one to find a qualitative picture of the spectrum. In particular, if the chirp vanishes at infinity, $v_{0}(x) \rightarrow 0$ at $|x|$ $\rightarrow \infty$, the greatest absolute value of $\lambda_{2}$ is approximately equal to

$$
\left|\lambda_{2}\right|_{\max }=\sqrt{\rho(\infty)}=\frac{1}{2}\left(\lambda_{1}-\lambda_{4}\right) .
$$

On the other hand, the smallest absolute values of $\left|\lambda_{2}\right|$ for large initial pulse must be very close to the absolute local minima of the potentials $\left|\lambda_{ \pm}(x)\right|$ and at the same time coincide with the amplitudes of the deepest solitons $\sqrt{\rho_{s}}$ [see Eq. (23)] in the trains produced by this pulse. This gives the relation

$$
\frac{1}{4}\left(\lambda_{1}-\lambda_{4}\right)^{2}-\left(\lambda_{1}-\lambda_{2}\right)\left(\lambda_{2}-\lambda_{4}\right)=\lambda_{2}^{2},
$$

which is satisfied by

$$
\lambda_{2}=\frac{1}{4}\left(\lambda_{1}+\lambda_{4}\right) \quad \text { or } \quad \lambda_{1}+\lambda_{4}=0 .
$$

Here the first solution is nonphysical because for symmetrical pulses we always have $\lambda_{4}=-\lambda_{1}$ but $\left|\lambda_{2}\right|_{\text {min }}$ must vary depending on the initial data. Thus, we conclude that in the asymptotic trains of solitons the constant parameters satisfy the relation

$$
\lambda_{4}=-\lambda_{1}
$$

and hence the possible values of $\lambda_{2}$ are located inside the intervals

$$
-\lambda_{\max }<\lambda_{2}<\lambda_{\min }^{\prime}, \quad \lambda_{\min }^{\prime \prime}<\lambda_{2}<\lambda_{\max },
$$

where $\lambda_{\max }=\sqrt{\rho(\infty)}$ and $\lambda^{\prime}, \lambda^{\prime \prime}\left(\lambda^{\prime}<\lambda^{\prime \prime}\right)$ correspond to the minima of the potentials $\left|\lambda^{ \pm}(x)\right|$. Solitons in the arising trains have the intensity given by

$$
\rho_{s}(x, t)=\lambda_{1}^{2}-\frac{\lambda_{1}^{2}-\lambda_{2}^{2}}{\cosh ^{2}\left[\sqrt{\lambda_{1}^{2}-\lambda_{2}^{2}}\left(x-2 \lambda_{2} t\right) / \varepsilon\right]} .
$$

Since the velocity of solitons (57) is equal to the values of $2 \lambda_{2}$ and at this asymptotic stage of the evolution we can neglect the "initial positions" of solitons, then the coordinate of the $n$th soliton at the moment $t$ is given by

$$
x=2 \lambda_{2}^{(n)} t,
$$

where $\lambda_{2}^{(n)}$ is the $n$th eigenvalue determined by Eq. (51). Hence, at the moment $t$ the soliton trains occupy the intervals

$$
-2 \lambda_{\max } t<x<2 \lambda_{\text {min }}^{\prime} t \quad \text { and } 2 \lambda_{\text {min }}^{\prime \prime} t<x<2 \lambda_{\max } t .
$$

Differentiation of Eq. (51) with respect to $\lambda$ yields the number of eigenvalues in the interval $(\lambda, \lambda+d \lambda)$,

$$
d n=f(\lambda) d \lambda=\left(\frac{1}{2 \pi \varepsilon} \oint \frac{\lambda-v_{0}(x) / 2}{\sqrt{\left[\lambda-v_{0}(x) / 2\right]^{2}-\rho_{0}(x)}} d x\right) d \lambda .
$$

This formula has a simple interpretation in terms of semiclassical quantization rules for the Hamiltonian (52). Namely, the "velocity of motion" between two turning points is given by

$$
\dot{x}=\frac{\partial H}{\partial p}=\frac{2 \lambda \sqrt{\left(\lambda-v_{0}(x) / 2\right)^{2}-\rho_{0}(x)}}{\lambda-v_{0}(x) / 2},
$$

and the calculation of the period of this oscillatory motion $T=\oint d x / \dot{x}$ and of the corresponding frequency $\omega=2 \pi / T$ shows that Eq. (60) can be written in the form

$$
\frac{d \lambda^{2}}{d n}=\varepsilon \omega(\lambda)=2 \pi \varepsilon\left(\oint \frac{d x}{\dot{x}}\right)^{-1},
$$


(a)

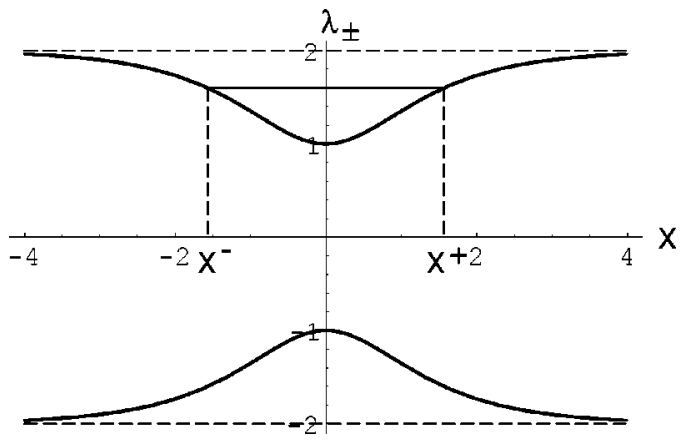

(b)

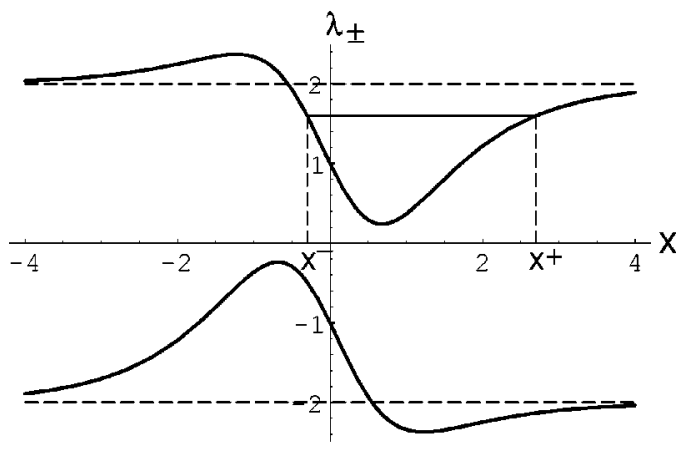

FIG. 4. Plots of initial potentials $\lambda_{ \pm}$[see Eq. (53)] as functions of $x$ for $\rho_{0}(x)$ and $v_{0}(x)$ given by Eqs. (63) and (64), (a) and (b), respectively. The turning points $x^{ \pm}$corresponding to the eigenvalue $\lambda=1.2$ are shown. The possible values of $\lambda$ are given by Eq. (65).

which means that the distance between neighboring eigenvalues of $\lambda^{2}$ is equal to the "quantum" $\varepsilon \omega$ proportional to the frequency $\omega$ of the classical motion. The rule (61) coincides with the quantum mechanical rule of semiclassical quantization with the Planck constant $\hbar$ replaced by $\varepsilon$ [33]. by

The number of solitons in the interval $(x, x+d x)$ is given

$$
d n=(1 / 2 t) f(x / 2 t) d x
$$

where $f(\lambda)$ is determined by Eq. (60). The amplitudes of these dark solitons are equal to $\lambda_{1}^{2}-\left(\lambda_{2}^{(n)}\right)^{2}$, and positive eigenvalues $\lambda_{2}^{(n)}>0$ correspond to solitons moving to the right and negative eigenvalues $\lambda_{2}^{(n)}<0$ to solitons moving to the left. Thus, the formulas obtained give the complete descriptions of the soliton trains arising from an initially large and smooth pulse.

Let us illustrate this theory by concrete examples and compare the theoretical predictions with the results of the direct numerical solution of the defocusing NLS equation (1).

At first we choose the initial distribution of intensity $\rho_{0}(x)$ in the form

$$
\rho_{0}(x)=\left(2-\frac{1}{\cosh x}\right)^{2}
$$

and the initial distribution of $v_{0}(x)$ as

$$
\text { either } \quad v_{0}(x)=0
$$
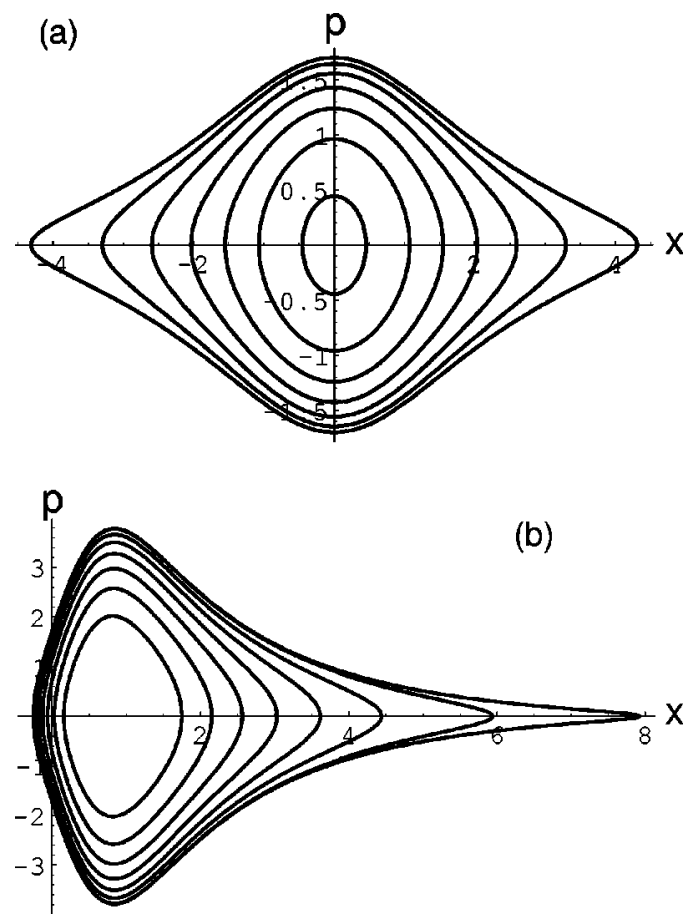

FIG. 5. Contours $H(p, x)=$ const $=\lambda^{2}$ in the phase plane $(x, p)$ of the mechanical system described by Hamiltonian (52) with $\rho_{0}(x)$ and $v_{0}(x)$ given by Eqs. (63) and $(64 \mathrm{a}, \mathrm{b})$ and for values of $\lambda$ determined by the quantization rule (51) with even $n$.

or

$$
v_{0}(x)=-4 \sinh x / \cosh ^{2} x
$$

The parameter $\varepsilon$ controlling the dispersion effects is chosen equal to $\varepsilon=0.2$. In Fig. 4 the plots of potentials (53) are shown for zero (a) and nonzero (b) initial velocities $v_{0}(x)$. The possible values of $\lambda$ are located inside the intervals (56) and they must satisfy the quantization rule (51) that selects contours $H(p, x)=$ const $=\lambda^{2}$ in the phase plane $(x, p)$. These contours corresponding to the even values of $n$ and $\lambda>0$ are depicted in Fig. 5 for zero (a) and nonzero (b) initial chirp. The dependence of $n$ on $\lambda>0$ is shown in Fig. 6 , where the lower curve corresponds to zero chirp and the

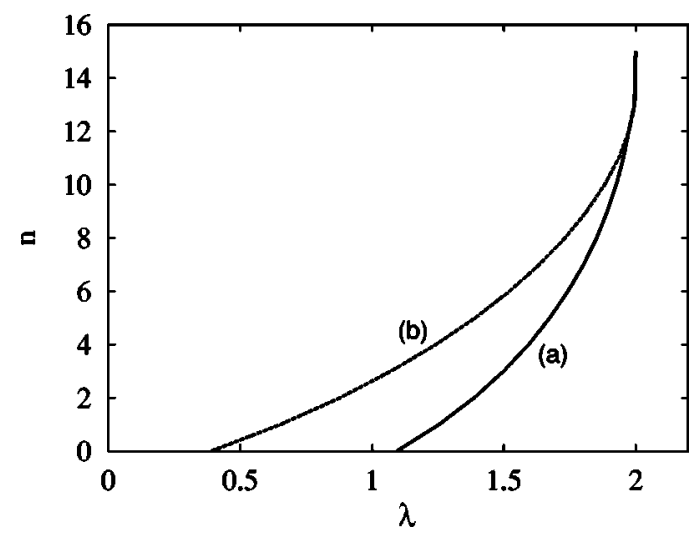

FIG. 6. The dependence of $n$ on $\lambda>0$ defined by Eq. (51) for $\rho_{0}(x)$ and $v_{0}(x)$ given by Eqs. (63) and (64). The lower curve corresponds to zero chirp (a) and the upper curve to nonzero chirp (b). 


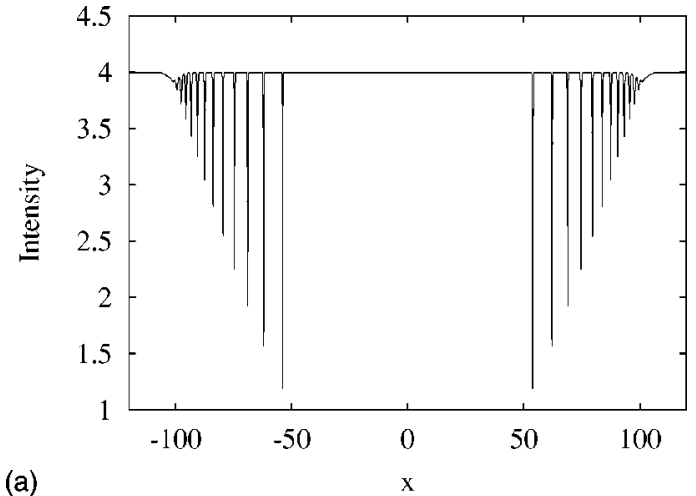

(a)

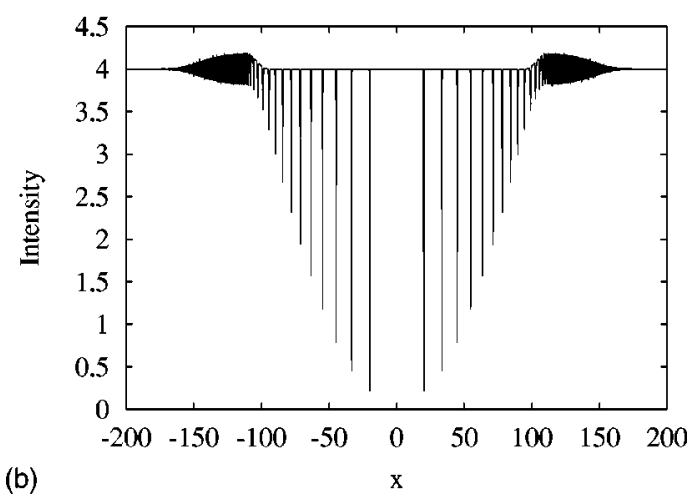

FIG. 7. Soliton trains obtained by numerical solution of the NLS equation (1) with $\varepsilon=0.2$ and initial conditions (63) and (64) with zero (a) and nonzero (b) initial chirp. Asymptotic distributions of intensity are shown as functions of space coordinate $x$ at the moment $t=25$.

upper curve to nonzero chirp. As we see, the chosen chirp diminishes the values of the eigenvalues $\lambda_{2}^{(n)}$, so that solitons arising in this case move slower than in the case of zero chirp.

We have solved the NLS equation (1) with the initial conditions (63) and (64) numerically and the results are shown in Fig. 7 again for zero (a) and nonzero (b) chirp. As one can see, the results are in excellent agreement with the predicted intervals. To make a comparison of the theory with numerical results clearer, we have calculated velocities of solitons in the trains and, according to Eq. (58), have taken halves of

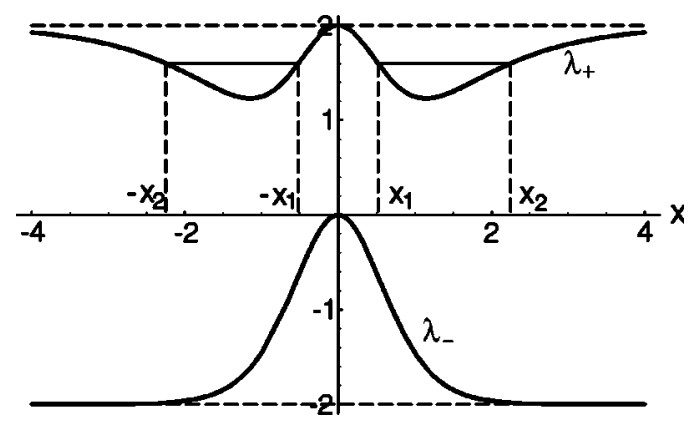

FIG. 8. The potentials $\lambda_{ \pm}(x)$ [see Eq. (53)] for the initial conditions (63) and (65).
TABLE I. Eigenvalues for the initial conditions (63) and (64).

\begin{tabular}{cccccc}
\hline \hline$n$ & \multicolumn{2}{c}{$v=0$} & & \multicolumn{2}{c}{$v=-\frac{4 \sinh x}{\cosh ^{2} x}$} \\
& & & & $\lambda_{\text {simulation }}$ \\
\cline { 2 - 3 } \cline { 5 - 6 } & $\lambda_{B S}$ & $\lambda_{\text {simulation }}$ & & $\lambda_{B S}$ & $\lambda_{n}$ \\
\cline { 5 - 6 } 0 & 1.094 & 1.078 & & 0.390 & 0.402 \\
1 & 1.254 & 1.243 & & 0.653 & 0.669 \\
2 & 1.387 & 1.379 & & 0.878 & 0.885 \\
3 & 1.499 & 1.494 & & 1.072 & 1.096 \\
4 & 1.594 & 1.592 & & 1.242 & 1.270 \\
5 & 1.674 & 1.676 & & 1.391 & 1.425 \\
6 & 1.743 & 1.749 & & 1.521 & 1.562 \\
7 & 1.802 & 1.812 & & 1.634 & 1.684 \\
8 & 1.852 & 1.866 & & 1.732 & 1.793 \\
9 & 1.892 & 1.913 & & 1.814 & 1.890 \\
10 & 1.926 & 1.954 & & 1.882 & 1.978 \\
11 & 1.953 & 1.989 & & 1.936 & 2.054 \\
\hline \hline
\end{tabular}

these velocities as values of $\lambda_{2}$ calculated numerically. We give in Table I the values of $\lambda_{2} \equiv \lambda_{B S}$ calculated from the Bohr-Sommerfeld quantization rule (51) and of $\lambda_{2}$ $\equiv \lambda_{\text {simulation }}$ calculated from velocities of the soliton trains. The agreement between the two methods of calculation is quite good. The difference does not exceed $3 \%$ and is maximal at very small $n$, where the accuracy of the semiclassical calculation of the eigenvalues cannot, generally speaking, be extremely high, and at large $n$, where the numerical estimate has poor accuracy because solitons here are not separated well enough from each other and their motion does not obey the formula (58) yet. The accuracy of asymptotic formulas increases with the decrease of $\varepsilon$. The number of solitons formed from a pulse with a given intensity is proportional to $\varepsilon$.

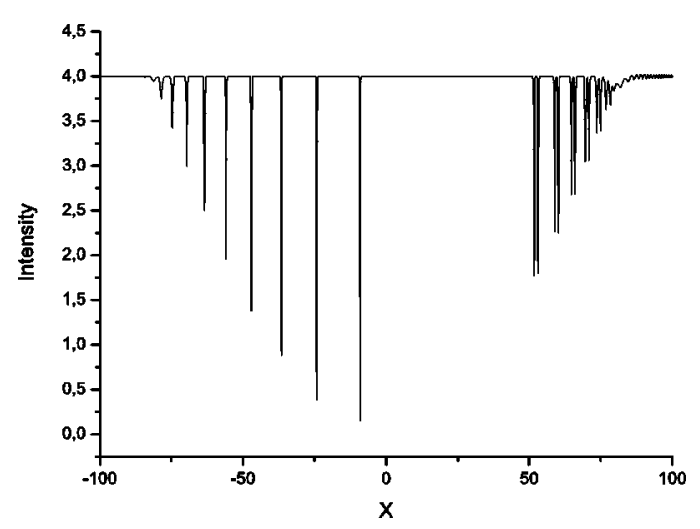

FIG. 9. Soliton trains obtained by numerical solution of the NLS equation (1) with $\varepsilon=0.2$ and initial conditions (63) and (65). The asymptotic distribution of intensity is shown as a function of space coordinate $x$ at the moment $t=20$. The train of usual solitons moves to the left and corresponds to negative eigenvalues in the potential $\lambda_{-}(x)$ and the train of twin solitons moves to the right and corresponds to positive slightly split eigenvalues in two-well potential $\lambda_{+}(x)$. 
TABLE II. Negative eigenvalues for the initial conditions (63) and (65).

\begin{tabular}{lcc}
\hline \hline$n$ & $\lambda_{B S}$ & $\lambda_{\text {simulation }}$ \\
\hline 0 & -0.2278 & -0.2280 \\
1 & -0.6073 & -0.6052 \\
2 & -0.9175 & -0.9155 \\
3 & -1.1775 & -1.1774 \\
4 & -1.39684 & -1.3998 \\
5 & -1.58083 & -1.5875 \\
6 & -1.73217 & -1.7432 \\
7 & -1.85163 & -1.8695 \\
8 & -1.93821 & -1.9647 \\
9 & -1.98889 & \\
\hline \hline
\end{tabular}

As a second example we shall consider the initial pulse with intensity (63) and more complicated chirp

$$
v_{0}(x)=\frac{d}{d x}\left(\frac{2 \sinh x}{\cosh ^{2} x}\right)=\frac{3-\cosh (2 x)}{\cosh ^{3} x}
$$

when the potentials (53) have the form shown in Fig. 8. The potential $\lambda_{-}(x)$ has usual one-well form with nondegenerate negative eigenvalues which can be calculated according to the semiclassical rule (51). However, the semiclassical eigenvalues of the two-well potential $\lambda_{+}(x)$ are double degenerate and by analogy with quantum mechanics they must be split into two very close levels with splitting proportional to the barrier penetration coefficient [33],

$$
\Delta \lambda_{2}^{(n)} \propto \exp \left(-\frac{1}{\varepsilon} \int_{-x_{1}}^{x_{1}}|p(x)| d x\right),
$$

so that the eigenvalues are given by

$$
\lambda_{2}^{(n) \pm}=\lambda_{2}^{(n)} \pm \frac{1}{2} \Delta \lambda_{2}^{(n)},
$$

where $\lambda_{2}^{(n)}$ correspond to the rule (51) and the integral in Eq. (66) is calculated at $\lambda=\lambda_{2}^{(n)}$. These close to each other eigenvalues must lead to the formation of "twin" solitons with very close values of their parameters. The intensity of the pulse obtained by the numerical solution of the NLS equation (1) with initial data (63) and (65) is shown in Fig. 9. Solitons moving to the left correspond to the nondegenerate eigenvalues and their velocities $2 \lambda_{2}^{(n)}$ agree quite well with ones calculated according to Eq. (51); see Table II. Twin
TABLE III. Positive eigenvalues for the initial conditions (63) and $(65) ; \lambda_{\text {simulation }}$ are calculated as arithmetic mean values for each couple of twin solitons.

\begin{tabular}{lcc}
\hline \hline$n$ & $\lambda_{B S}$ & $\lambda_{\text {simulation }}$ \\
\hline 0 & 1.329 & 1.311 \\
1 & 1.498 & 1.489 \\
2 & 1.637 & 1.635 \\
3 & 1.749 & 1.757 \\
4 & 1.838 & 1.857 \\
5 & 1.907 & 1.941 \\
\hline \hline
\end{tabular}

solitons moving to the right correspond to slightly split eigenvalues of the spectral problem with two-well potential $\lambda^{+}(x)$ and the mean velocity of each couple agrees well enough with the semiclassical rule (51); see Table III.

\section{CONCLUSION}

The semiclassical approach developed in this paper permitted us to consider in some detail the evolution of pulses governed by the defocusing NLS equation. Two characteristic stages of evolution have been considered-the formation of the dissipationless shock after the wave breaking point and asymptotic soliton trains formed eventually from a large initial pulse. It has been shown that the semiclassical approach to the calculation of the eigenvalues of the problem (2) yields a simple and effective description of the asymptotic stage of the evolution of an initially large and smooth pulse in the weak dispersion limit. The results show that the arising solitons can be slowed down by the initial chirp, but it cannot prevent the formation of solitons from a large pulse due to dispersion effects. As typical examples, the usual soliton trains and twin soliton trains are considered. Their characteristic features are controlled by the initial conditions, what may occur useful for applications. The theoretical predictions are confirmed by numerical simulations.

\section{ACKNOWLEDGMENTS}

A.M.K. and B.A.U. are grateful to the staff of Instituto de Física Teórica, UNESP (where this work was started) for kind hospitality. The authors were partially supported by FAPESP (Brazil). A.M.K. thanks also RFBR (Grant No. 0101-00696), R.A.K. thanks CNPq (Brazil), and B.A.U. thanks CRDF (Grant No. ZM2-2095) for partial support.
[1] Yu.S. Kivshar and B. Luther-Davies, Phys. Rep. 298, 81 (1998).

[2] M.G. Forest and K.T.-R. McLaughlin, J. Nonlinear Sci. 7, 43 (1998).

[3] O.C. Wright, M.G. Forest and K.T.-R. McLaughlin, Phys. Lett. A 257, 170 (1999)

[4] G.B. Whitham, Linear and Nonlinear Waves (WileyInterscience, New York, 1974).
[5] A.M. Kamchatnov, Nonlinear Periodic Waves and Their Modulations-An Introductory Course (World Scientific, Singapore, 2000).

[6] A.V. Gurevich and L.P. Pitaevskii, Zh. Éksp. Teor Fiz. 65, 590 (1973) [Sov. Phys. JETP 38, 291 (1973)].

[7] P.D. Lax and C.D. Levermore, Commun. Pure Appl. Math. 36, 253 (1983); 36, 571 (1983); 36, 809 (1983).

[8] A.V. Gurevich, A.L. Krylov and G.A. El, Zh. Éksp. Teor. Fiz 
1011797 (1992) [Sov. Phys. JETP 74, 957 (1992)].

[9] F.R. Tian, Commun. Pure Appl. Math. 46, 1093 (1993).

[10] G.A. El and A.L. Krylov, Phys. Lett. A 203, 77 (1995).

[11] Y. Kodama, SIAM (Soc. Ind. Appl. Math.) J. Appl. Math. 59, 2162 (1999).

[12] S. Jin, C.D. Levermore, and D.W. McLaughlin, Commun. Pure Appl. Math. 52, 613 (1999).

[13] F.-R. Tian and J. Ye, Commun. Pure Appl. Math. 52, 655 (1999).

[14] G.V. Potemin, Usp. Matem. Nauk, 43211 (1988) [Russ. Math. Surveys 43, 252 (1988)].

[15] O.C. Wright, Commun. Pure Appl. Math. 46, 423 (1993).

[16] V.I. Karpman, Phys. Lett. A25, 708 (1967).

[17] V.I. Karpman, Nonlinear Waves in Dispersive Media (Pergamon, Oxford, 1975).

[18] M.S. Alber, G.G. Luther, and J.E. Marsden, Nonlinearity 10, 223 (1997).

[19] A.M. Kamchatnov, R.A. Kraenkel, and B.A. Umarov, Phys. Lett. A 287, 223 (2001).

[20] Y. Kodama and S. Wabnitz, Opt. Lett. 20, 2291 (1995).

[21] D. Krökel, N.J. Halas, G. Giuliani, and D. Grischkowsky, Phys. Rev. Lett. 60, 29 (1988).
[22] M. Chen, M.A. Tsankov, J.M. Nash, and C.E. Patton, Phys. Rev. Lett. 70, 1707 (1993).

[23] A.N. Slavin, Yu.S. Kivshar, E.A. Ostrovskaya and H. Benner, Phys. Rev. Lett. 82, 2583 (1999).

[24] V.E. Zakharov and A.B. Shabat, Zh. Eks. Teor. Fiz. 641627 (1972) [Sov. Phys. JETP 37, 823 (1973)].

[25] A.M. Kamchatnov and R.A. Kraenkel, J. Phys. A 35, L13 (2002).

[26] S.J. Alber, in Nonlinear Processes in Physics, edited by A.S. Fokas et al. (Springer, Berlin, 1993), p. 6.

[27] M.G. Forest and J.E. Lee, in Oscillation Theory, Computation, and Methods of Compensated Compactness, edited by C. Dafermos, J.L. Erickson, D. Kinderleher, and M. Slemrod, IMA Volumes on Mathematics and its Applications 2 (Springer, New York, 1986).

[28] M.V. Pavlov, Teor. Mat. Fiz. 71, 351 (1987) [Theor. Math. Phys. 71584 (1987)].

[29] S.P. Tsarev, Izv. Akad. Nauk SSSR Ser. Matem. 54, 1048 (1990) [Math. USSR Izv. 37, 397 (1991)].

[30] J.C. Bronski, Physica D 97, 376 (1996).

[31] P.D. Miller, Physica D 152-153, 145 (2001).

[32] J.C. Bronski, Physica D 152-153, 163 (2001).

[33] L.D. Landau and E.M. Lifshitz, Quantum Mechanics (Pergamon, London, 1959). 